\title{
Cone-Jet Analytical Extension of Taylor's Electrostatic Solution and the Asymptotic Universal Scaling Laws in Electrospraying
}

\author{
Alfonso M. Gañán-Calvo \\ Escuela Superior de Ingenieros, Universidad de Sevilla, Reina Mercedes s/n, 41012 Sevilla, Spain
}

(Received 21 March 1997)

\begin{abstract}
An analytical cone-jet solution for the electrohydrodynamic atomization of liquids has been found for an asymptotic model assuming an infinitely long and thin emitted jet. Universal expressions for the emitted electric current, jet shape, charge distribution, surface charge, and other essential electrohydrodynamic quantities are obtained as functions of the liquid properties and the emitted liquid flow rate. The agreement with published experiments is good. [S0031-9007(97)03566-7]
\end{abstract}

PACS numbers: $47.65 .+\mathrm{a}, 47.55 .-\mathrm{t}$

In this Letter we report a successful analytical approach to the complex and multidisciplinary problem of the electrohydrodynamic spraying of liquids. For sufficiently large electrification levels [1,2], this spraying method uses the strong, local electric forces appearing at a charged liquid-gas interface to provoke liquid ejections in the form of capillary liquid threads from the tip of conelike protrusions, giving rise to extremely fine charged aerosols with vast applications in science and technology. Here we consider the most relevant regime, the so-called cone-jet mode [3] (see Fig. 1), achieved when a conical liquid meniscus is formed at the tip of an electrified capillary needle for a range of electric potentials [1-4]. A steady, thin but robust liquid thread with a typical diameter from several microns to nanometers (which eventually breaks up into remarkably homogeneous size droplets) is emitted from the apex of the conical meniscus when a steady constant liquid flow rate $Q$ is supplied through the needle. Briefly outlined, our model approach consists of a basic electrostatic conical solution plus an infinitely long and thin jet issuing from its apex. The electrohydrodynamic process of liquid and charge emission is solved, and the emitted electric current, jet shape, charge distribution, etc. are, for the first time, analytically determined. Except for the notation, the reader mostly interested in applications may skip the following up to the Results paragraph.

Basic electrostatic solution.-In the absence of liquid emission (no jet), Taylor [2] obtained an exact, infinite electrostatic solution in spherical coordinates $(R, \theta)$ (see Fig. 1) consisting of an infinite, perfectly conical equilibrium shape with semiangle $\theta_{T}=0.86027432 \ldots$ The electric potential outside this electrified cone is given by

$$
\Phi_{T}(R, \theta)=\left(\frac{2 \gamma}{\varepsilon_{0}}\right)^{1 / 2} \mathcal{D}_{0} Q_{1 / 2}(\theta) R^{1 / 2}
$$

where

$$
\mathcal{D}_{0}=\left[\tan \left(\theta_{T}\right) Q_{1 / 2}^{\prime}\left(\theta_{T}\right)\right]^{-1 / 2},
$$

$\gamma, \varepsilon_{0}$, and $Q_{1 / 2}$ stand for the liquid-surrounding gas surface tension, electrical permittivity of vacuum, and
Legendre modified function of order $1 / 2$, respectively. The symbol ' means derivative respect to $\theta$. The liquid dielectric constant $\varepsilon_{i}$ is absent in this result since it deals with an electrostatic solution of a perfectly conducting liquid, for which the inner electric field is null.

Electric cone-jet model. - Consider now a very thin and very long charged liquid jet issuing from the tip of Taylor's cone. Even in the case of a leaky dielectric (not perfectly conducting) liquid [5], if it moves slowly enough to allow for electric relaxation, i.e., in time scales large as compared to the electric relaxation time $t_{e}=\varepsilon_{i} / K$ (where $K$ stands for the liquid electrical conductivity), the liquid bulk is quasineutral and the charges stay at the surface [5-8]. The electric field in the liquid $\mathbf{E}^{i}$ is then very small compared to the outer one $\mathbf{E}$. We call this a "quasielectrostatic/perfectly conducting" (QEPC) limit, for which the surface charge $\sigma_{s}=\varepsilon_{0} E_{n}-\varepsilon_{i} E_{n}^{i}$ can be expressed simply as $\sigma_{s} \simeq \varepsilon_{0} E_{n}$, where $E_{n}$ and $E_{n}^{i}$ are the normal components of $\mathbf{E}$ and $\mathbf{E}^{i}$, respectively, at the liquid surface. The role of $\varepsilon_{i}$ is then negligible.

In our QEPC limit, Taylor solution $\Phi_{T}$ for the outer field is modified by the appearance of a new term, say $\Phi_{G}$, owing to the jet. As long as our geometry allows superposition, sufficiently far away from the point $R=0$, we can search for a solution of the problem as $\Phi=$ $\Phi_{T}+\Phi_{G}$. We will use the natural representation of the electric field in spherical coordinates, given in terms of

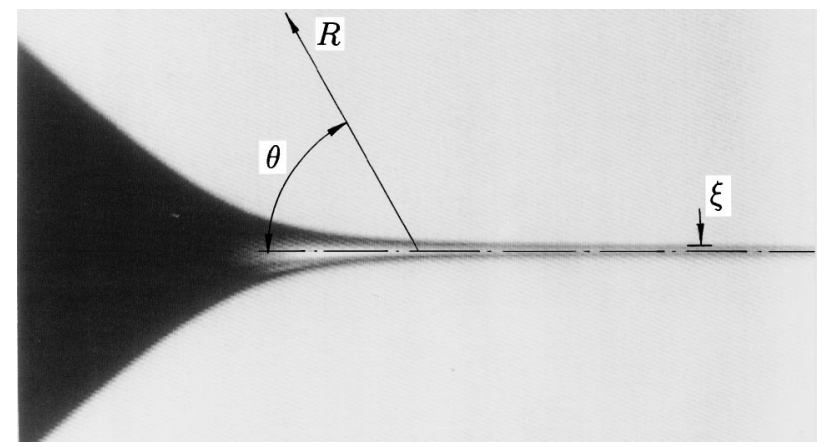

FIG. 1. Steady cone-jet configuration and spherical coordinates system. 
Legendre functions, to write $\Phi_{G}$ as a series of the form

$$
\Phi_{G}(R, \theta)=\sum_{\nu} D_{\nu}\left[Q_{\nu}(\theta)+C_{\nu} P_{\nu}(\theta)\right] R^{\nu},
$$

where $Q_{\nu}$ and $P_{\nu}$ stand for the Legendre functions of order $\nu$, and $\{\nu\}$ is a certain infinite sequence of numbers which satisfies the requirements of completeness of the series. This sequence will be consistently found, and $D_{\nu}$ and $C_{\nu}$ will be solved as part of the problem. The representation in spherical coordinates is useful to derive relations at the cone. Let us write the cone surface as $\theta=\theta_{T}+\zeta$, where $\zeta$ is a function of $R$ representing the departure from Taylor's conical shape $\theta=\theta_{T}$ owing to the presence of the jet (space charge effect). First, the potential decay along the cone surface is several orders of magnitude smaller than the one at the jet, owing to the large transversal section of the cone $[8,9]$. Therefore, its surface can be considered equipotential. Second, surface tension must be balanced by the electrostatic force. Assuming $\zeta \ll \theta_{T}$, one can linearize these two conditions around $\theta=\theta_{T}$ and obtain, after some algebra, the expression

$$
\zeta=-\sum_{\nu}\left(\frac{\varepsilon_{0}}{2 \gamma}\right)^{1 / 2} D_{\nu} \frac{Q_{\nu}\left(\theta_{T}\right)+C_{\nu} P_{\nu}\left(\theta_{T}\right)}{Q_{1 / 2}^{\prime}\left(\theta_{T}\right)} R^{-1 / 2+\nu}
$$

where and

$$
C_{\nu}=-\frac{\frac{2 Q_{\nu}^{\prime}\left(\theta_{T}\right)}{\tan \theta_{T}}-[(\nu-1 / 2)(\nu+3 / 2)+\beta] Q_{\nu}\left(\theta_{T}\right)}{\frac{2 P_{\nu}^{\prime}\left(\theta_{T}\right)}{\tan \theta_{T}}-[(\nu-1 / 2)(\nu+3 / 2)+\beta] P_{\nu}\left(\theta_{T}\right)}
$$

$\beta=\frac{1+\tan ^{2} \theta_{T}}{\tan ^{2} \theta_{T}}+\frac{2 Q_{1 / 2}^{\prime \prime}\left(\theta_{T}\right)}{\tan \theta_{T} Q_{1 / 2}^{\prime}\left(\theta_{T}\right)}=0.2596483 \ldots$.

Furthermore the jet surface, located around the axis $\theta=\pi$, can be represented as $\xi=(\pi-\theta) R$, where $\xi$ stands for the local radius of the jet assuming $\xi \ll R$

(Fig. 1). The local geometry of the jet is then a very slowly varying cylinder. This suggests the use of more familiar expressions of the electric fields at the jet surface [i.e., when $\theta \rightarrow \pi$ in expression (3)]. In fact, one may define

$$
A_{\nu}=-\left(\cos (\nu \pi)+\frac{2 C_{\nu}}{\pi} \sin \nu \pi\right) D_{\nu},
$$

$$
\begin{aligned}
B_{\nu}=-\left(\cos (\nu \pi)+\frac{2 C_{\nu}}{\pi} \sin (\nu \pi)\right) D \nu / & {\left[\gamma_{E}+\boldsymbol{\Psi}(\nu+1)-\ln 2\right]\left(\cos (\nu \pi)+\frac{2 C_{\nu}}{\pi} \sin (\nu \pi)\right)-\frac{\pi}{2} \sin (\nu \pi) } \\
& \left.+C_{\nu} \cos (\nu \pi)\right]
\end{aligned}
$$

in terms of $D_{\nu}$ and $C_{\nu}$ (where $\gamma_{E}$ and $\boldsymbol{\Psi}$ are the Euler constant and the digamma function, respectively). Thus, the series $A(R)=\sum_{\nu} A_{\nu} R^{\nu}$ and $B(R)=\sum_{\nu} B_{\nu} R^{\nu}$ allow one to write expressions for the electric fields $\left(E_{n}, E_{s}\right)$ at the jet surface (as in cylindrical coordinates) simply as

$$
\begin{gathered}
E_{n}=A / \xi, \\
E_{s}=E_{T}+\frac{d}{d R}[A \ln (\xi / R)+B],
\end{gathered}
$$

for $\xi \ll R$, where

$$
E_{T}=\left(\frac{2 \gamma}{\varepsilon_{0}}\right)^{1 / 2} \frac{\pi \mathcal{D}_{0}}{4} R^{-1 / 2}
$$

Functions $A(R)$ and $B(R)$ represent a charge distribution located at the axis of symmetry and the nonsingular part of the correction to Taylor's solution, respectively.

Jet hydrodynamics. - Since there is a tangential electric field $E_{s}$ pointing in the axial direction, there is a momentum exerted by the electric stress on the surface with value $\tau_{s}=\sigma_{s} E_{s}$. In the limit of an "infinitely" thin jet, this momentum is rapidly diffused in the radial direction by viscous stresses throughout the jet transversal section, and the velocity profile becomes almost flat with value $v=Q /\left(\pi \xi^{2}\right)[7,8,10]$. In this limit, the liquid momen- tum balance can be expressed as

$$
\frac{d}{d R}\left[P+\frac{1}{2} \rho \frac{Q^{2}}{\pi^{2} \xi^{4}}\right]=\frac{2 \tau_{s}}{\xi},
$$

where $P$ stands for the liquid pressure. Since the pressure jump across the jet surface is in a large extent balanced by surface tension (in addition to the electrostatic and the polarization forces [8]) its value is of the order of $\gamma / \xi$. Therefore, $P \sim \gamma / \xi$ can be neglected vs the kinetic energy $\rho Q^{2} /\left(2 \pi^{2} \xi^{4}\right)$ for very thin jets $\xi \rightarrow 0$ [8]. The capillary equation can be henceforth excluded from the analysis.

Finally, the electric current is driven by both the surface charge motion and the bulk electric conduction $[7,8]$ :

$$
I=2 \pi \xi \sigma_{s} \frac{Q}{\pi \xi^{2}}+K \pi \xi^{2} E_{s} .
$$

Notice that while the first term becomes dominant downstream along the jet, where the jet radius $\xi$ becomes very small, the second one is dominant close to the cone apex, where the jet is thicker. In particular, bulk electric conduction is several orders of magnitude larger than the surface electric convection at the cone, where a very small radial electric field in the liquid bulk provokes the charge migration towards the apex [9]. 
Universal scaling.-First, using the parameters $\left\{\rho, K, \gamma, \varepsilon_{0}\right\}$ one can define several characteristic quantities: a characteristic flow rate $Q_{0}=\rho K \gamma^{-1} \varepsilon_{0}^{-1}$, an electric current $I_{0}=\varepsilon_{0}^{1 / 2} \gamma \rho^{-1 / 2}$, a distance $d_{0}=$ $\left(\pi^{-2} \gamma \varepsilon_{0}^{2} \rho^{-1} K^{-2}\right)^{1 / 3}$, and an electric field $E_{0}=$ $\left(2 \gamma \varepsilon_{0}^{-1} d_{0}^{-1}\right)^{1 / 2}$. For a given liquid, it has been shown that $Q_{0}, d_{0}$, and $I_{0}$ are actually of the order of the minimum flow rate, jet diameter, and emitted electric current, respectively, that can be obtained by electrospraying $[6,11,12]$. Introducing the nondimensional flow rate $Q=Q / Q_{0}$, and the number $\mathcal{L}_{0}=\ln Q^{1 / 2}$, let us define (i) a typical jet radius $R_{0}=d_{0} \mathcal{Q}^{1 / 2}$, (ii) an axial distance $L_{0}=d_{0} \mathcal{Q} \mathcal{L}_{0}$, (iii) a typical value of the normal field at the jet surface $E_{n 0}=E_{0} \mathcal{L}_{0}^{-1 / 2}$, (iv) the same for the axial field, $E_{s 0}=E_{0}\left(Q \mathcal{L}_{0}\right)^{-1 / 2}$, and (v) a typical potential variation along the jet $\Phi_{0}=E_{0} L_{0}$. Note that in the asymptotic limit $Q \gg 1$, the characteristic axial length of the jet $L_{0}$ is very large as compared to the jet radius $R_{0}$. In addition, $\mathcal{L}_{0}$ becomes a large number.

With these definitions, one can write Eqs. (8), (10), and (11) as a rather simple system of nondimensional universal equations in terms of the nondimensional quantities $z=R / L_{0}, \quad f=\xi / R_{0}, \quad a=A /\left(E_{n 0} R_{0}\right)=$ $A /\left(E_{s 0} L_{0} \mathcal{L}_{0}^{-1}\right), e_{s}=E_{s} / E_{s 0}$, and $e_{s 0}=\left(E_{T}+\dot{B}\right) / E_{s 0}$, with the nondimensional current $I=I /\left(8 I_{0}^{2} \mathcal{Q} / \mathcal{L}_{0}\right)^{1 / 2}$ :

$$
\begin{aligned}
e_{s}=e_{s 0}-\dot{a}, \quad \dot{f} & =-2 a f^{3} e_{s}, \\
\frac{e_{s} f^{2}}{2}+\frac{\pi a}{f^{2}} & =I .
\end{aligned}
$$

Terms of the order $\mathcal{L}_{0}^{-1}$ and smaller have been neglected in system (12).

When $z \rightarrow \infty$ (i.e., close to the jet breakup), surface charge advection becomes dominant (i.e., $\pi a / f^{2} \rightarrow I$ ), since the liquid jet must eventually break up and the liquid domain is no longer continuous. In addition, the tangential electric field at the surface $e_{s}$ approaches $e_{s 0}$ since the potential decay in the radial direction from the surface, of the order $E_{n 0} R_{0}$, is negligible as compared to the potential decay owing to the external field, of the order $E_{s 0} L_{0}$. When such conditions at $z \rightarrow \infty$ are imposed, the asymptotic solution of system (12) is given by

$$
\begin{gathered}
f_{\infty}=\frac{z^{-1 / 8}}{\left(4 I \mathcal{D}_{0}\right)^{1 / 4}}, \quad a_{\infty}=\frac{I^{1 / 2} z^{-1 / 4}}{2 \pi \mathcal{D}_{0}^{1 / 2}}, \\
e_{s \infty}=\frac{\pi}{4} \mathcal{D}_{0} z^{-1 / 2} .
\end{gathered}
$$

The solution of system (12) for $z \sim O(1)$ is now consistently found in terms of power series of the form

$$
\kappa=\kappa_{\infty}\left(1+\sum_{n=1}^{\infty} \alpha_{n} z^{-3 n / 4}\right),
$$

where $\kappa$ stands for $f, a$, or $e_{s}$. The potential $\Phi_{G}$ is then given by expression (3) with $\{\nu\}=\{1 / 2-$ $3 n / 4\}_{(n=1,2, \ldots, \infty)}$. System (12), together with expressions (4) allow the complete and consistent calculation of the series by solving the terms $\left\{\alpha_{n}\right\}_{f, a, e_{s}}$ as functions of the eigenvalue $I$, the nondimensional electric current.
Electric problem around $z=0$. - Series (14) are absolutely convergent outside a sphere of radius $R^{*}$ (which is a function of $I$ ) around the origin $z=0$, and diverges inside. The charge distribution $a(z)$ at the axis of symmetry inside that sphere, univocally determined from the values of the electric field and potential at the convergence radius, can be represented in terms of positive powers of $z$ such as $a=\sum_{n=0}^{\infty} \gamma_{n} z^{n}$. This series is solved using the overlapping region of absolute convergence of both series. Linearizing the expression $\Phi=\Phi_{T}+\Phi_{G}$ around $\theta=\theta_{T}$ and invoking (3) and (4), one obtains that the value of the potential at the cone surface is equal to 0 . Since the solution at the jet's side is regular at $z=R^{*}$, while the cone turns into a cusp close to the convergence radius of the outer series [see Fig. 2(a)], Eqs. (12) are still valid inside the sphere from the jet's side up to the point where $f \rightarrow \infty$. Thus, the solution of the problem is given by the value of $I$ for which the potential becomes 0 when $f$ goes to $\infty$ inside the sphere. One finds $I \simeq 1.5$, after

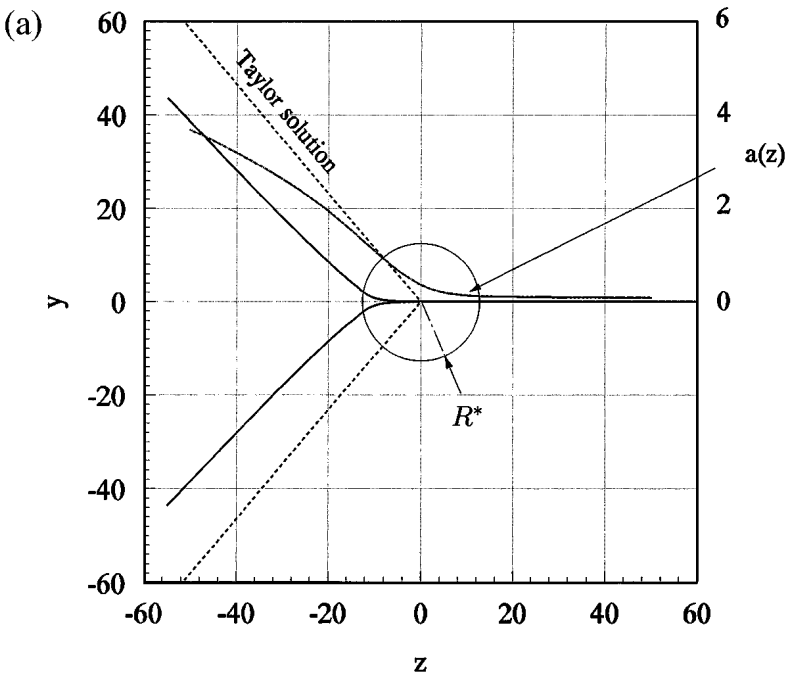

(b)

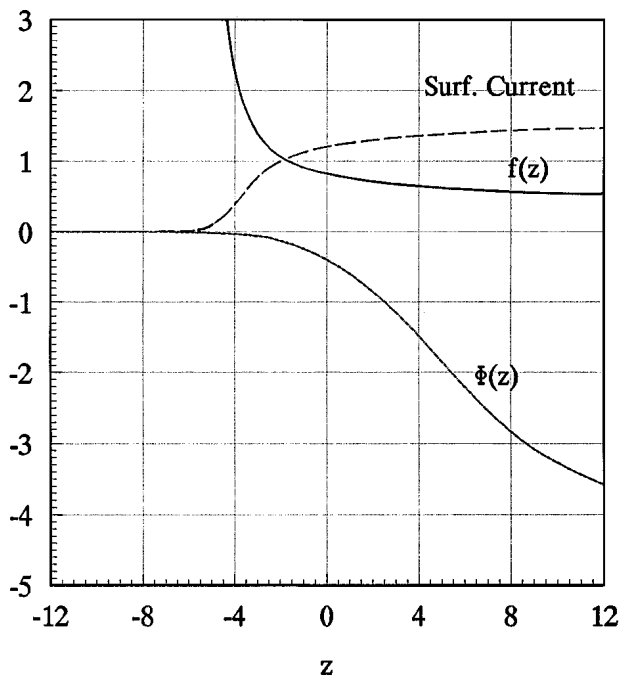

FIG. 2. (a) Cone-jet shape, and charge distribution $a(z)$ at the axis. Also plotted, the Taylor cone (---). (b) Jet shape, surface advection current, and potential decay along the jet. 

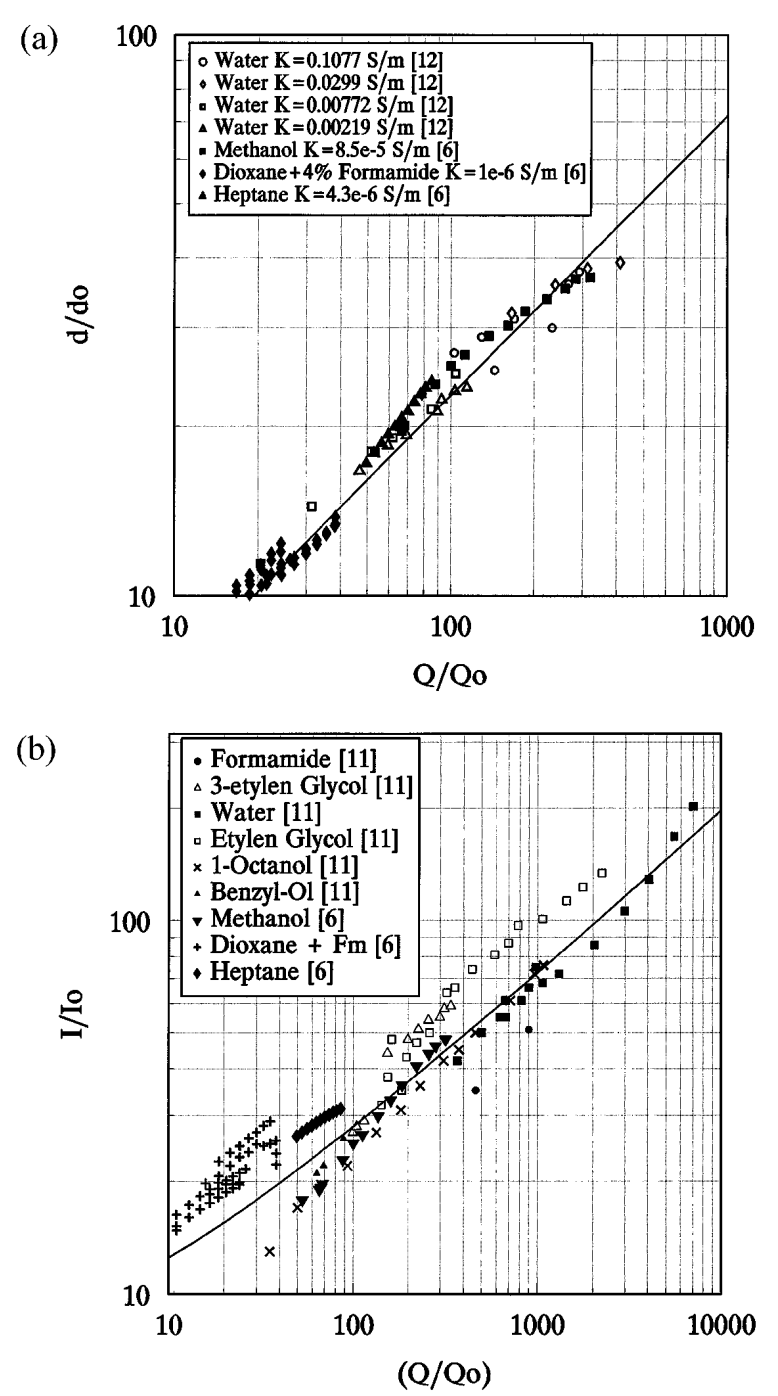

FIG. 3. (a) Scaling of the measured current for three representative liquids from [6], and data from [11] for $Q / Q_{0} \geq 30.0$. The asymptotic universal scaling is given by a solid line. (b) Scaling of the experimentally measured droplet diameter for the same liquids from [6], and for water-sucrose solutions, given in [12]. The theoretical universal scaling for the droplet diameter given by a solid line.

integrating Eqs. (12) with a convergence radius of the outside series $R^{*} \simeq 12.5$. The inner solution gives the transition from the jet to the cone, and shows how the electric current changes from dominant Ohmic conduction at the cone to surface charge advection at the jet [8].

Results. - The nondimensional charge distribution at the axis of symmetry $a(z)$ and the shape of the cone are given in Fig. 2(a). Other results of interest, for example, the jet shape $f$, the surface advection current, and the potential decay at the jet axis, are plotted in Fig. 2(b).

In terms of physical quantities, the total electric current and droplet diameter given by our model are

$$
I=4.25\left[Q K \gamma / \ln \left(\frac{Q}{Q_{0}}\right)^{1 / 2}\right]^{1 / 2}=4.25\left(\frac{Q K \gamma}{\mathcal{L}_{0}}\right)^{1 / 2},
$$

$$
d=2 \times 1.89 R_{0} f_{b}=3.78 \pi^{-2 / 3} Q^{1 / 2}\left(\frac{\rho \varepsilon_{0}}{\gamma K}\right)^{1 / 6} f_{b},
$$

$f_{b}$ being the nondimensional radius of the jet at the breakup point. 1.89 stands for the Rayleigh most probable jet to droplet relationship, valid for electrospray [3]. However, droplet size cannot be exactly obtained from this analysis since there is no breakup region in the asymptotic model. In reality, the jet breaks up in most cases at a point located roughly from $z \sim 10$ to $z \sim 100$ depending on the liquid viscosity. Since the jet shape changes as slowly as $z^{-1 / 8}$, one may estimate an average value $f_{b} \simeq 0.6$ within maximum errors of the order of $25 \%$, even below the experimental uncertainties in some cases. These universal asymptotic scalings are compared with results from some experimental studies $[6,11,12]$, using many different liquids with permittivities spanning from $\varepsilon_{i}=1.9 \varepsilon_{0}$ to $\varepsilon_{i}=111.0 \varepsilon_{0}$. The electric current and droplet diameter are given in Figs. 3(a) and 3(b), respectively.

The present analytical results suggest that both the electric current and the droplet diameter are independent of the liquid polarity. Liquids with large polarities usually present large conductivities too, resulting in large experimental $Q / Q_{0}$ values, which might have led to attribute to the liquid polarity the role actually played by $\mathcal{L}_{0}=\ln Q / Q_{0}$ in the experimental correlations $[6,11,12]$. The present analytical results also explain why the chargeto-mass ratio $Q / I$ shows a power law approximately inversely proportional to $d$, noted by many authors (see [12]) but unexplained before. Finally, this analytical model serves as a local solution close to the apex of a real electrified cone, where the emission takes place. While this region is local enough, the influence of the needle-electrode potential difference in the emitted current and droplet size is small, as shown in most published experiments $[6,11,12]$.

This work is supported by the Spanish Comisión Interministerial de Ciencia y Tecnología, Project No. PB931181.

[1] J. Zeleny, Phys. Rev. 10, 1 (1917).

[2] G. I. Taylor, Proc. R. Soc. London 280, 383 (1964).

[3] M. Cloupeau and B. Prunet-Foch, J. Electrost. 22, 135 (1989).

[4] C. Pantano, A.M. Gañán-Calvo, and A. Barrero, J. Aerosol Sci. 25, 1065 (1994).

[5] D. A. Saville, Annu. Rev. Fluid Mech. 29, 27 (1997).

[6] A. M. Gañán-Calvo, J. Dávila, and A. Barrero, J. Aerosol Sci. 28, 249 (1997).

[7] J. R. Melcher and E. P. Warren, J. Fluid Mech. 47, 127 (1971).

[8] A. M. Gañán-Calvo, J. Fluid Mech. 335, 165 (1997).

[9] D. P. H. Smith, IEEE Trans. Ind. Appl. 22, 527 (1986).

[10] A. J. Mestel, J. Fluid Mech. 274, 93 (1994).

[11] J. Fernández de la Mora and I. G. Loscertales, J. Fluid Mech. 260, 155 (1994).

[12] D.-R. Chen, D. Y.H. Pui, and S. L. Kaufman, J. Aerosol Sci. 26, 963 (1995). 